\title{
A More Effective Method of Extracting the Characteristic Value of Pulse Wave Signal Based on Wavelet Transform
}

\author{
Xuanwei Zhang, Yazhou Shang, Daoxin Guo, Tianxia Zhao, Qiuping Li, Xin'an Wang \\ The Key Laboratory of Integrated Microsystems, Peking University Shenzhen Graduate School, Shenzhen, China \\ Email: xuanweiz0413@sz.pku.edu.cn
}

How to cite this paper: Zhang, X.W., Shang, Y.Z., Guo, D.X., Zhao, T.X., Li, Q.P. and Wang, X.A. (2016) A More Effective Method of Extracting the Characteristic Value of Pulse Wave Signal Based on Wavelet Transform. J. Biomedical Science and Engineering, 9, 9-19.

http://dx.doi.org/10.4236/jbise.2016.910B002

Received: May 26, 2016

Accepted: September 20, 2016

Published: September 23, 2016

\begin{abstract}
Pulse wave contains human physiological and pathological information. Different people will exhibit different characteristics, and hence determining the characteristic points of the pulse wave of human physiological health makes sense. It is common that we extract the characteristic value of pulse wave signal with the method based on wavelet transform on a small scale, and then determine the locations of the characteristic points by modulus maxima and modulus minima. Before determining characteristic value by detecting modulus maxima and modulus minima, we need to determine every period of the pulse wave. This paper presents a new kind of adaptive threshold determination method which is more effective. It can accurately determine every period of the pulse wave, and then extract characteristic values by modulus maxima and modulus minima in every period of the pulse wave. The method presented in this paper promotes the research utilizing pulse wave on health life.
\end{abstract}

\section{Keywords}

Pulse Wave, Wavelet Transform, Adaptive Threshold, Characteristic Values

\section{Introduction}

The contraction-relaxation cycle in the heart makes the blood in the heart chamber entering the aorta in the form of waves. The waves start to propagate from the root of the aorta artery along each artery, and these waves are pulse waves [1]. The pulse wave is the external reflection of the heart and blood vessels' important state information, and any change of our body system state will affect the pulse system. When blood flows through the body's capillaries, the capillary blood volume begins to change because of 
heart's fluctuations. Because the changes of the blood volume reflect the changes of blood vessels, we use infrared photoelectric pulse sensor to record changes of blood volume, and blood volume changing over time produce pulse wave [2].

The article is about processing and analyzing the pulse waveform collected from fingers. The pulse waveform collected from fingers is shown in Figure 1.

In Figure 1, A and B represent main peak and main valley respectively. $\mathrm{C}$ and $\mathrm{D}$ represent the dicrotic wave peak and dicrotic valley. They are closely related to cardiovascular physiological information [3]. Pulse wave (main wave, tidal waves, dicroticwave etc.) contains a wealth of physiological information [4]. These characteristic values extracted can provide much valuable diagnostic information, and then they have an important medical value [5].

There are many traditional methods to extract characteristic values. For example, Zhao Zhiqiang [6] proposed differential threshold method. He took differential operations on rising and falling edges of the pulse wave signal to highlight its characteristics. Then he determined the characteristic points according to the direction of differential operators. This approach has achieved some success, but there are some limitations. It may have some false detection or missed features.

The wavelet transform is a mathematical method developed rapidly in recent years. Its important feature of time-frequency localization analysis is that the time window

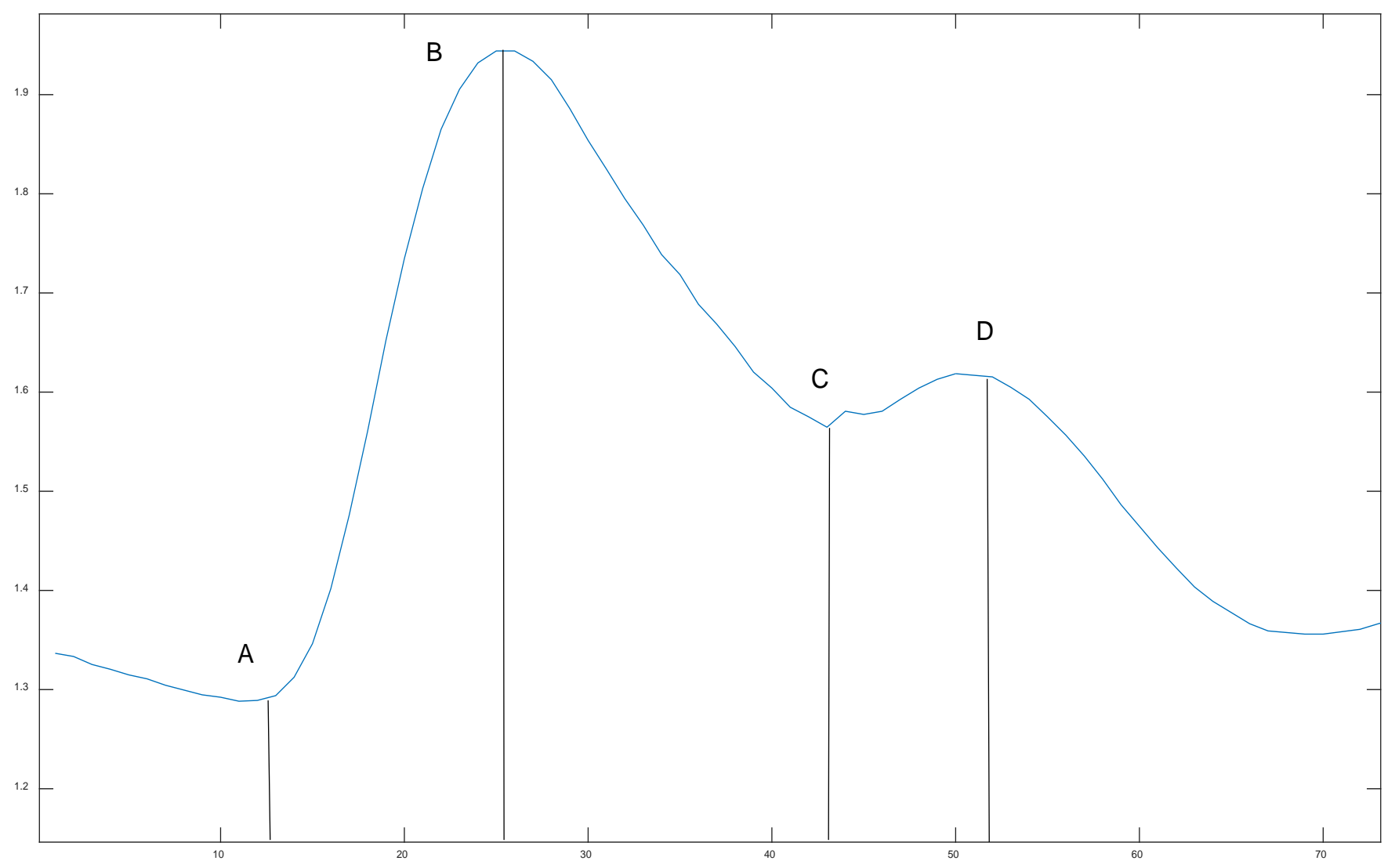

Figure 1. The pulse waveform collected from fingers. 
and frequency window can be changed. Therefore, wavelet transform is adaptive. Compared to other mathematical methods, wavelet transform is very suitable for nonstationary signals such as pulse wave [7]. Although researchers have applied wavelet transform to processing and analyzing the pulse wave in recent years. Their effect is not very good. At the same time these methods do not have a universal type.

To solve these problems, this paper proposes a new approach to extract characteristic points based on wavelet transform. The method can automatically select the referenced modulus maxima and the referenced modulus maxima are accurate even if waveforms are complex. Then we can accurately extract characteristic values.

\section{Pulse Wave Signal Preprocessing}

Proper analysis of the pulse wave is based on the pulse wave signal containing a small amount of noise and clear waveform. However, the acquisition of the pulse wave signal is easily affected by a variety of the influence of interference, so that the pulse wave collected contains a lot of noise. Therefore, reducing noise is particularly necessary. Signal denoising uses the method based on wavelet transform and multi-resolution analysis. The method can characterize the local signal information in the time domain and frequency domain, making the effect of denoising more obvious.

\subsection{Denoising Principle}

After wavelet decomposition of 4 levels, noisy pulse wave signal is decomposed into high frequency detail coefficients and low frequency approximation coefficients. Signal denoising actually suppresses unwanted signal portion and retain the part of the useful signal. Based on experience, the noisy signal corresponding to the relevant part of the high-frequency detail is removed, and then the remaining part is reconstructed to obtain new useful signal [8].

Specific processes of signal denoising are shown in Figure 2.

\subsection{Process and Results of Denoising}

Pulse wave wavelet decomposition of 4 levels is showed Figure 3.

In Figure 3, d1, d2, d3, d4 are the $1^{\text {st }}$ to $4^{\text {th }}$ level detail coefficients of wavelet decomposition, a 4 is the $4^{\text {th }}$ level approximate coefficients. As we can see, high frequency noise

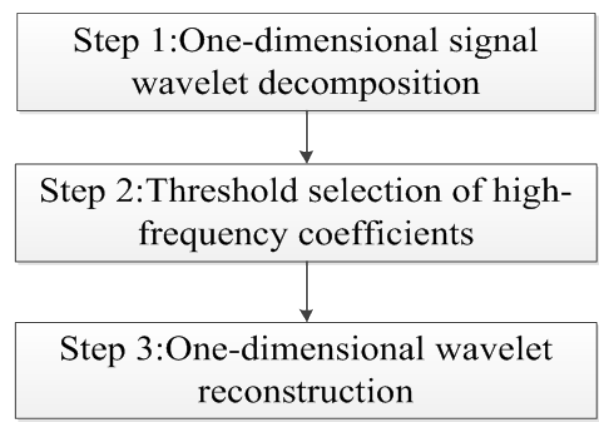

Figure 2. Specific processes of signal de-noising. 

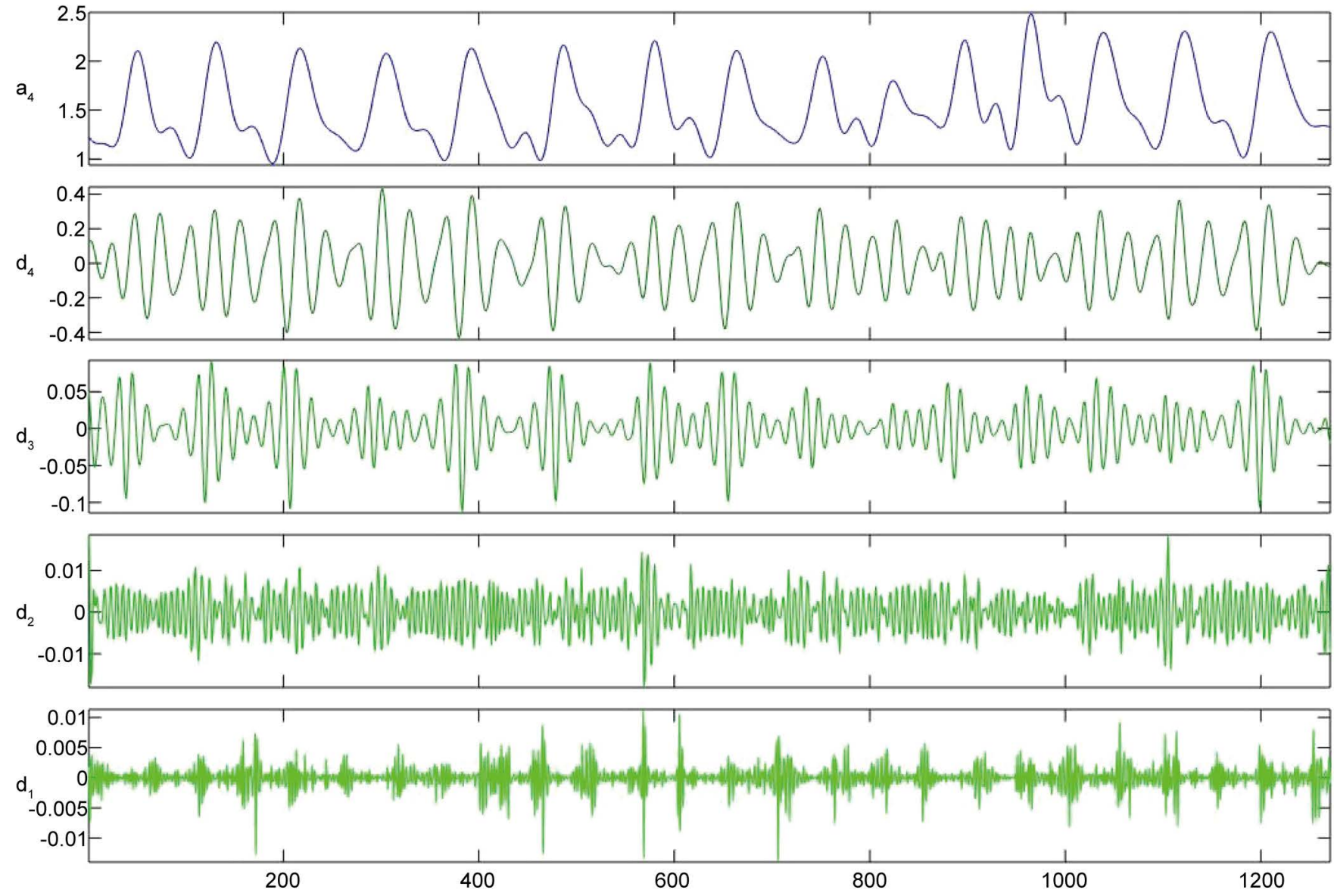

Figure 3. Pulse wave wavelet decomposition of 4 levels.

signals focus on the first layer and the second layer detail coefficients. So we suppress high frequency noise signals on the first layer and the second layer detail coefficients, and then reconstruct pulse wave signal.

There are three denoising methods by threshold, such as Mandatory threshold denoising, the default threshold denoising and given soft (or hard) threshold denoising. In method of Mandatory threshold denoising, the high-frequency coefficients of the wavelet decomposition structure are set to zero. This method is simple and the reconstructed signal is relatively smooth, but it is extremely easy to lose useful components of signal. In method of given soft (or hard) threshold denoising, it needs subjective experience and is also very tedious and time-consuming. So in the end we choose the default threshold denoising. Its threshold generated by the system, the coefficients which is less than the threshold are set to zero, and then the remaining coefficients are taken to reconstructing signal [9]. The results of denoising results are shown in Figure 4.

\section{Extracting Characteristic Values}

\subsection{Locating the Characteristic Points of Pulse Wave}

The core of the existing location methods of the characteristic points of pulse wave is to 


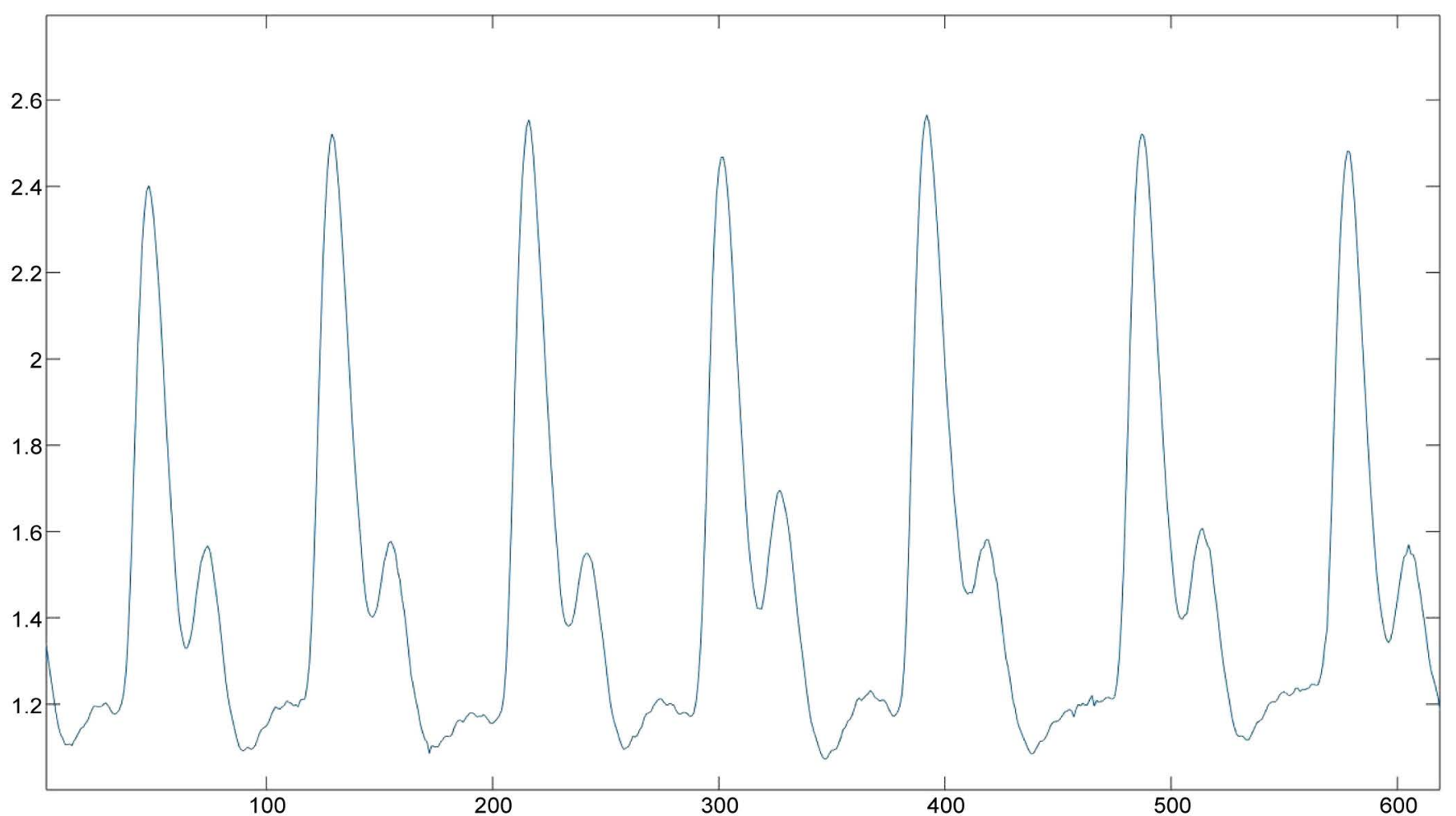

(a)

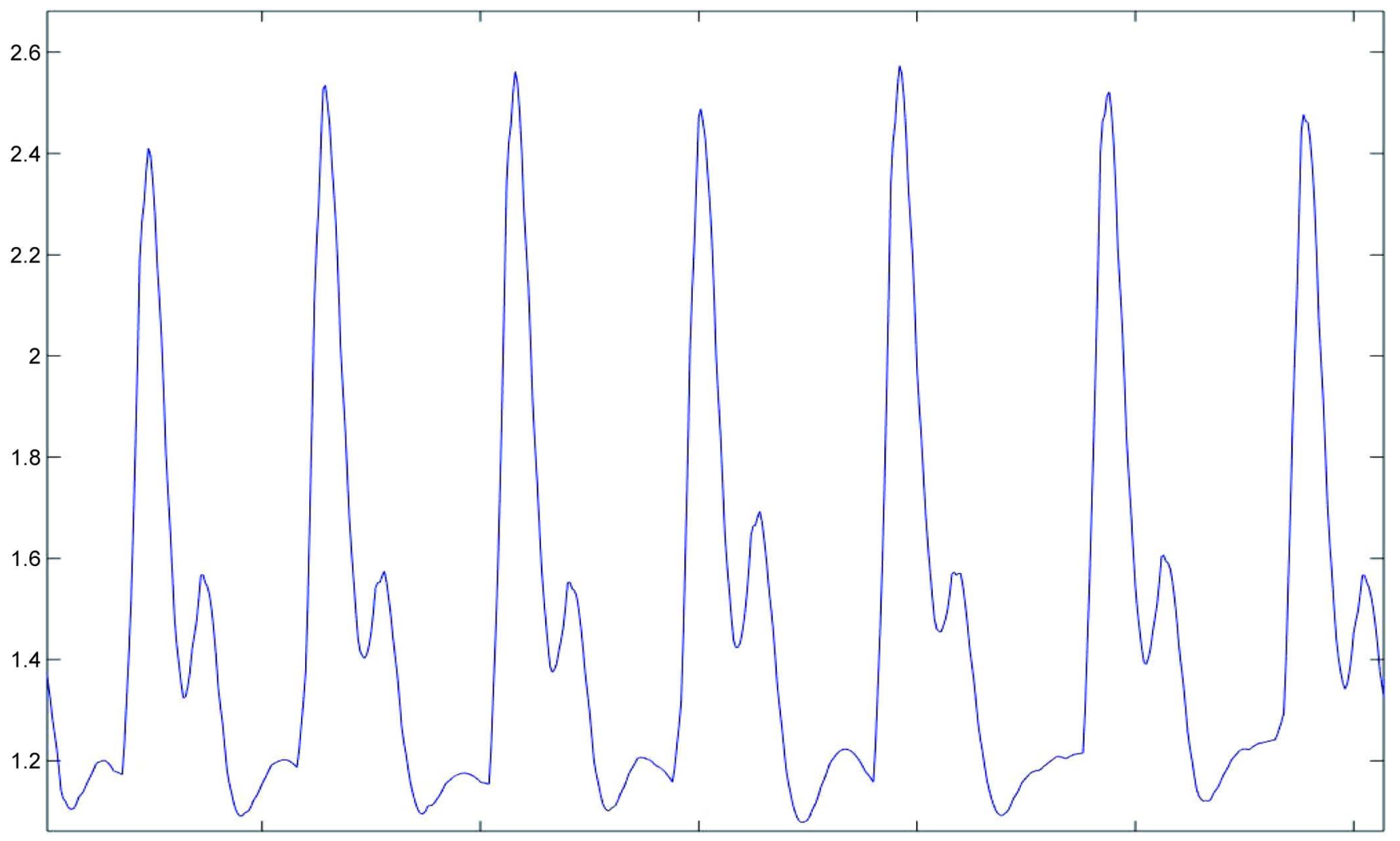

(b)

Figure 4. (a) The noisy pulse waveform; (b) The denoising pulse waveform. 
search for the wavelet transform modulus minima or modulus maxima within a scale or several scales. Then we connect these modulus minima or modulus maxima, the intersection of the straight line and the horizontal axis or locations of modulus values are locations of the characteristic points of pulse wave. Some researchers have a certain degree of error correction. However, these corrections cannot locate accurately characteristic points. The main reason is that the modulus minima or the modulus minima as reference points cannot be determined. Aiming at this problem, this paper proposes an adaptive determination threshold method. The determination of the modulus minima or the modulus minima as reference points makes further improvements through the method. Then we can accurately locate the position of characteristic points.

In a pulse wave period, the process of locating the characteristic points of pulse wave is as follows:

Step 1, we make wavelet decomposition of 4 levels after de-noising signal, and then the distribution of modulus values are shown Figure 5.

Step 2, after determining the largest modulus maximum during a pulse wave period, we look for adjacent modulus minimum value. Then we connect the modulus maximum and the modulus minimum, and find the intersection of the straight line and the horizontal axis, and then in the vicinity of the waveform corresponding to the intersection, we find the lowest point that is considered to be the location of valley value during the period.

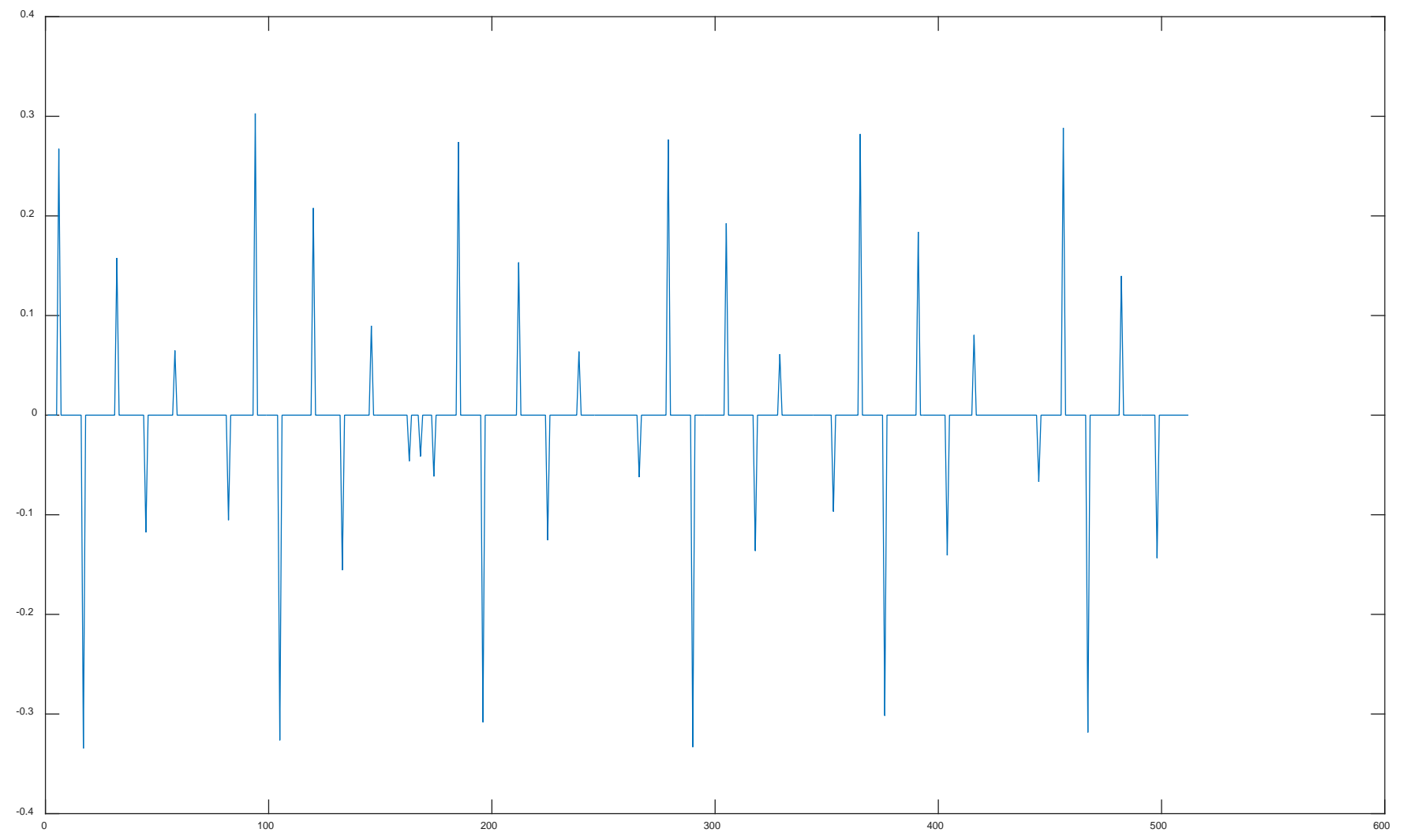

Figure 5. The distribution of modulus values. 
Step 3, by the same method, we find three intersections of three straight lines and the horizontal axis. And then in the vicinity of the waveform corresponding to each intersection we find the location of peak value, the location of dicrotic wave peak, the location of dicrotic wave valley.

Step 4, in the end, we get four characteristic points in a pulse wave period: the main peak, the main valley, dicrotic wave peak, dicrotic wave valley, which are shown in Figure 6.

Specific processing flow is shown in Figure 7.

\subsection{The Principle of the Adaptive Threshold Determination Method}

As we all know, the key point of determining the location of eigenvalues is to find the maximum modulus maxima position, and then we can find the locations of the corresponding feature points.

This paper has taken an effective method for accurately locating characteristic points, which is an adaptive threshold determination method. Therefore, we can precisely determine each pulse wave period.

Specific process is showed as follows:

First, we find this signal module maxima and minima. $A$ is representative of the largest module maximum. Then $\mathrm{A}$ is divided into $\mathrm{N}$ parts. Each size is $\mathrm{D}=\mathrm{A} / \mathrm{N}$. In theory, $\mathrm{N}$ is the bigger, the effect is the better. But the amount of calculation cannot be too large. In this paper, we select $\mathrm{N}=15$.

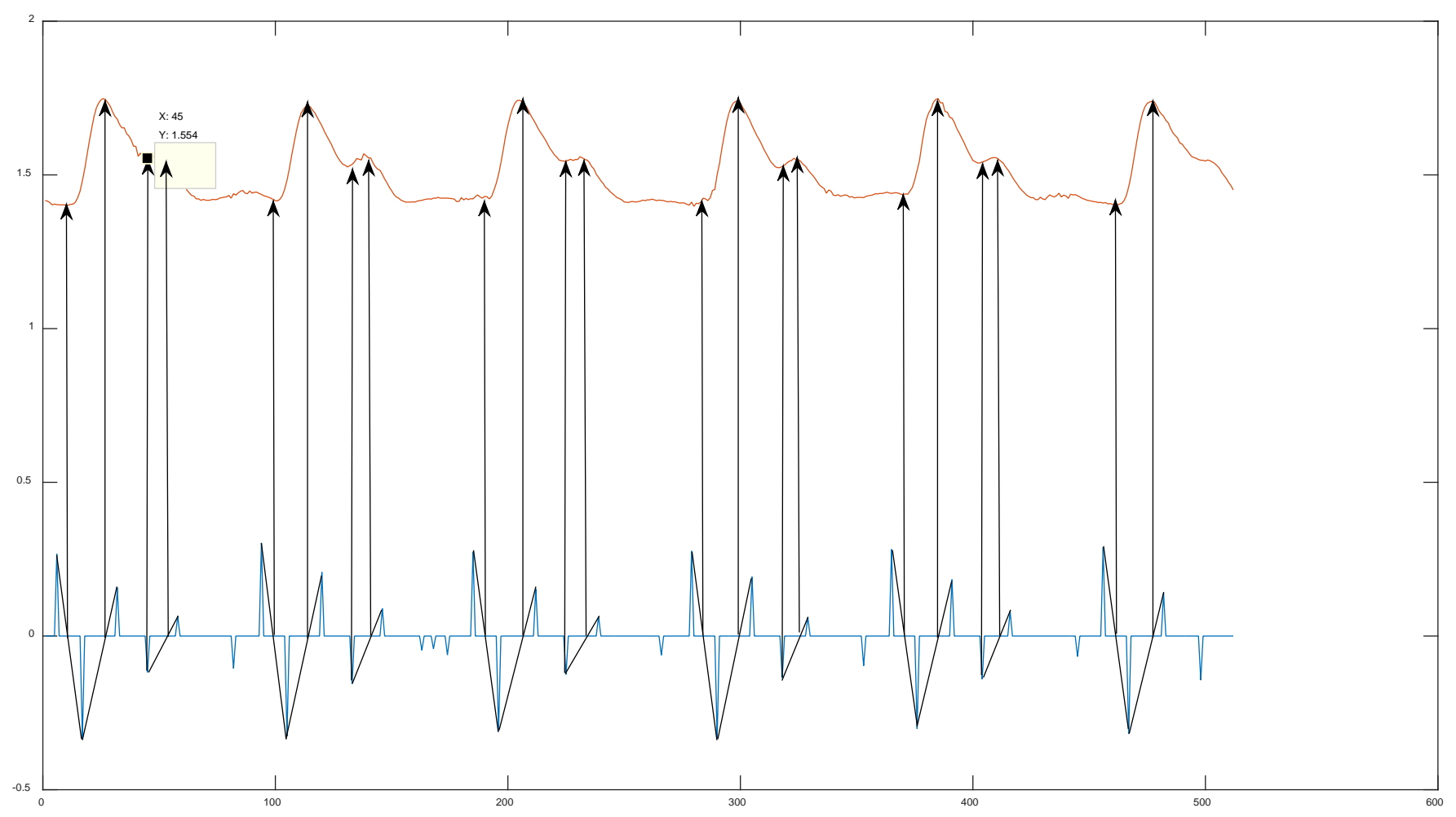

Figure 6. Characteristic points of pulse wave. 
making wavelet decomposition of 4 levels after signal de-noising

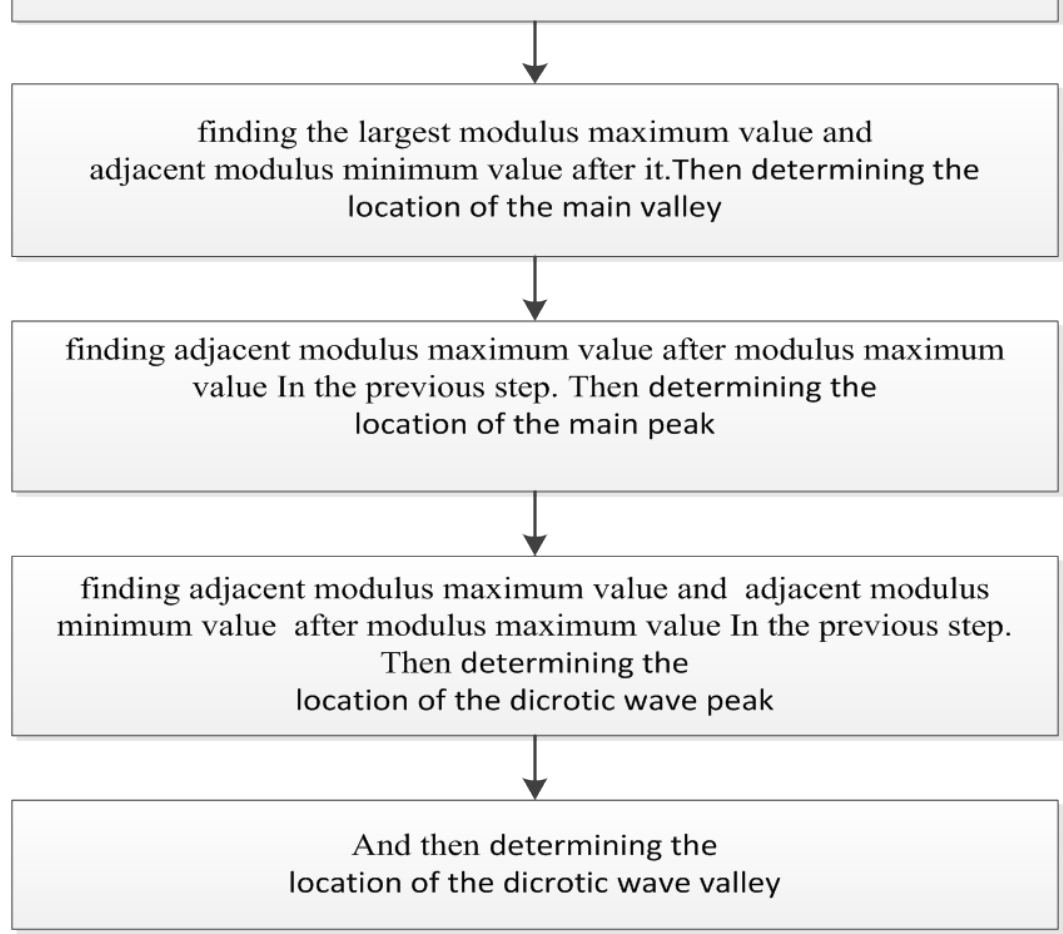

Figure 7. Location of the characteristic points of pulse wave.

Second, the largest module maximum is divided into $\mathrm{N}$ parts, so there are many threshold values. The first threshold value is $\mathrm{D}$, the second threshold value is $2{ }^{\star} \mathrm{D} \ldots \ldots$. the threshold value is $\mathrm{M} * \mathrm{D}, \mathrm{M}$ ranges from $0 \sim \mathrm{N}-1$.

Third, we record module maxima exceeding the threshold in case of each threshold. With different threshold values, module maxima exceeding the threshold values are different. The minimum distances between the locations of the module maxima are also different.

Fourth, when the minimum distance in the case of the threshold value is more than 2 times of the minimum distance in the case of the threshold value. We think the threshold value is what we need. Thus, we can determine each period of the pulse wave by the threshold. Then we can determine the characteristic points in each period.

Threshold determination process is shown in Figure 8.

\section{Simulation Results and Discussion}

The paper is using MATLAB simulation program. The collected pulse wave calibrates characteristic points with the method. Calibration results are shown in Figure 9.

As we can see from Table 1, the locations of the four characteristic values (the main peak, the main valley, dicrotic wave peak and dicrotic wave valley) in each pulse wave period are more precise. Thus the method proposed in the paper is a solution to the inaccurate positioning characteristic values in the conventional methods. 


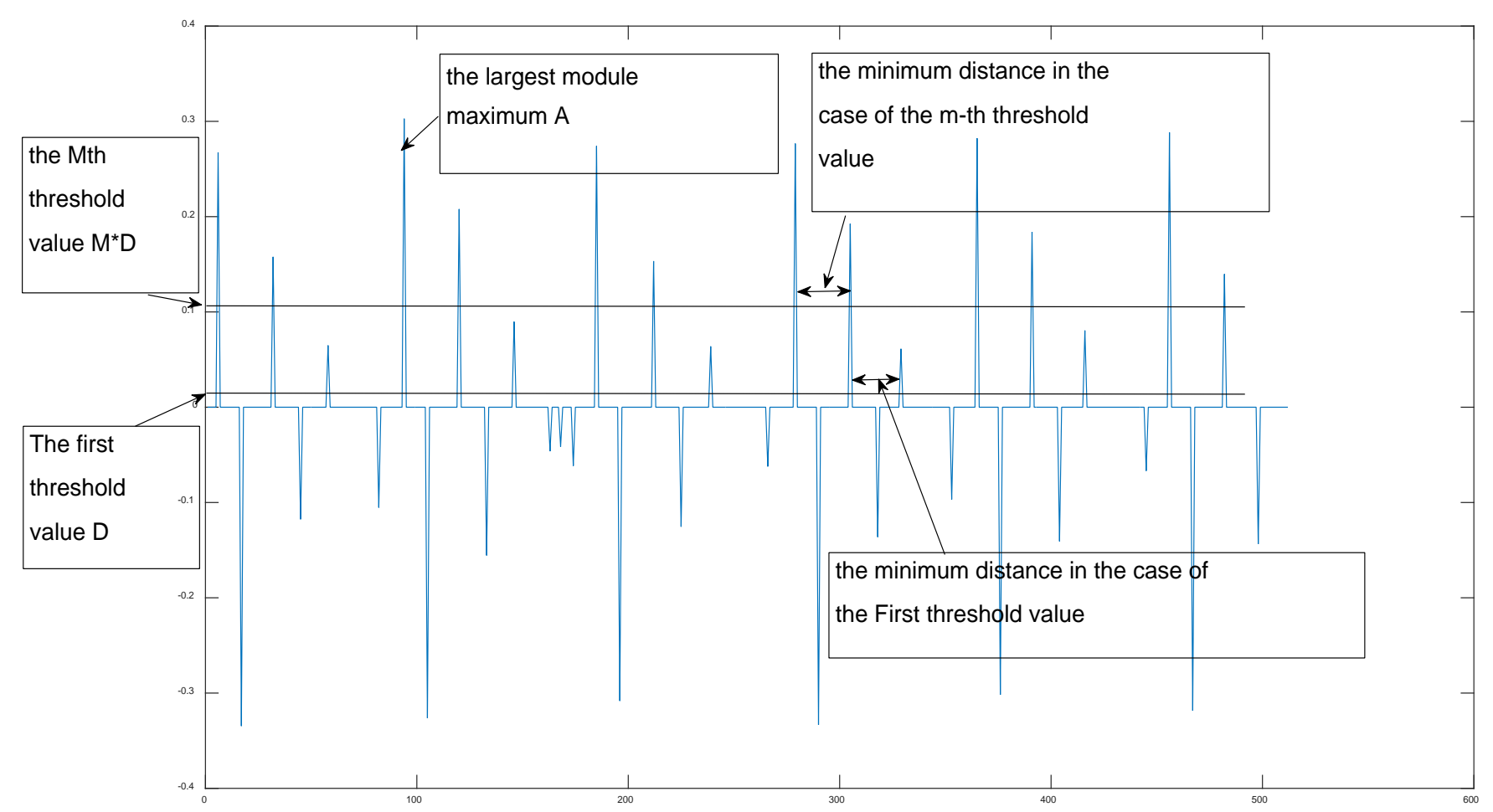

Figure 8. Threshold determination process.

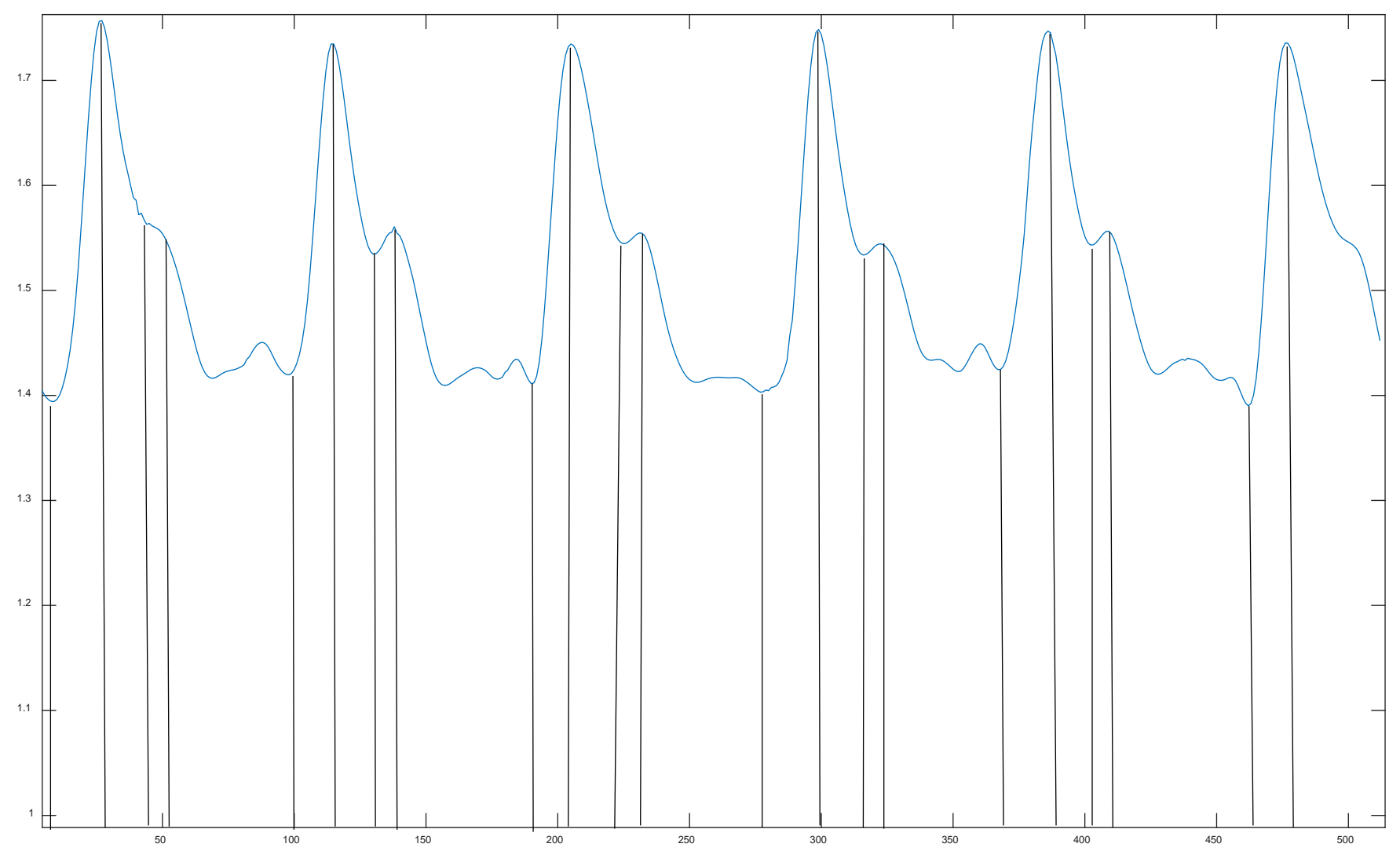

Figure 9. Calibration results. 
Table 1. Comparison between results and actual values.

\begin{tabular}{|c|c|c|c|c|c|c|}
\hline \multicolumn{2}{|c|}{ Order of the pulse wave } & 1 & 2 & 3 & 4 & 5 \\
\hline \multirow{3}{*}{ Results } & The main valley & 1.395 & 1.44 & 1.416 & 1.403 & 1.425 \\
\hline & The dicrotic valley & 1.572 & 1.535 & 1.544 & 1.544 & 1.543 \\
\hline & The dicrotic peak & 1.573 & 1.561 & 1.555 & 1.534 & 1.556 \\
\hline \multirow{3}{*}{ Actual values } & The main peak & 1.757 & 1.735 & 1.735 & 1.749 & 1.747 \\
\hline & The dicrotic valley & 1.572 & 1.535 & 1.544 & 1.534 & 1.543 \\
\hline & The dicrotic peak & 1.573 & 1.561 & 1.555 & 1.544 & 1.556 \\
\hline
\end{tabular}

The paper is only a preliminary study. the conclusion is also obtained in the case of few samples. The locations of the four characteristic values will be affected by others factor, such as breathing, equipment interference and so on. For example, when someone has atrial fibrillation, shapes of pulse wave is various and then the locations of the four characteristic values is also difficult. In the aspect of clinical medicine, we need a lot of experiments and medically confirmed.

\section{Conclusions}

In this paper, we apply wavelet transform to the pulse signal. And on this basis, we find the location of each characteristic point.

The method proposed in the paper is concise and practical. It is easy to be understood and provides a basis for accurately finding characteristic values of the pulse wave of time domain. At the same time, the paper also provides a solid foundation for the clinical research future of the pulse wave.

\section{Acknowledgements}

First and foremost I offer my sincerest gratitude to my supervisor, Professor Wang, who has supported me though out my thesis with his patience and knowledge. I am grateful to Yazhou Shang and Ran Peng for their encouragement and practical advice. This research is supported by R\&D projects of Shenzhen government by NO. JCYJ20140417144423206 and JCYJ20150331102721193.

\section{References}

[1] Di, N.-Q. and Xie, M. (2007) Pulse Waveform Interpretation. Chinese Journal of Information on Traditional Chinese Medicine, 6, 3-4.

[2] Nitzan, M., khanokh, B. and Slovik, Y. (2002) The Difference in Pulse Transit Time to the toe and Finger Measured by Photo-Plethysmography. Physiol Meas, 23, 85-93. http://dx.doi.org/10.1088/0967-3334/23/1/308

[3] Tang, M.-Y., Li, K. and Ma, X.-T. (2010) Research on Pulse Wave Signal and Time-Domain Feature Extraction Algorithm. Computer and Modernization, 176, 16-22. 
[4] Qu, N.Q. and Xie, M.Z. (2007) The Definition of Pulse Wave. Chinese Journal of Information on Tradition Chinese Medicine, 14, 3-4.

[5] Doherty, P., Grabowski, M., Lukaszewicz, W., et al. (2003) Towards a Framework for Approximate Ontologies. Fundamenta Informaticae, 57, 147-165.

[6] Zhao, Z.Q., Zheng, G.W., Shen, W., et al. (2013) Research on Pulse Wave Signal Noise Reduction and Feature Point Identification. Electronic Design Engineering, 21, 57-60.

[7] Daubechies, I. (1990) The Wavelet Transform, Time-Frequency Locatization and Signal Analysis. IEEE Transactions on Information Theory, 36, 961-1001.

http://dx.doi.org/10.1109/18.57199

[8] Kivanc Mihcak, M., Kozintsev, I., Ramchandran, K. and Moulin, P. (1999) Low-Complexity Image Denoising Based on Statistical Modeling of Wavelet Coefficients. IEEE Signal Processing Letters, 6, 300-303. http://dx.doi.org/10.1109/97.803428

[9] Zhang, X., Wang, H.Y. and Li, B.J. (2010) Powerline Interference Denoising Algorithm Analysis of ECG Signals Based on Wavelet Transform. Computer Measurement \& Control, 18, 902-905.

\section{Submit or recommend next manuscript to SCIRP and we will provide best service} for you:

Accepting pre-submission inquiries through Email, Facebook, LinkedIn, Twitter, etc.

A wide selection of journals (inclusive of 9 subjects, more than 200 journals)

Providing 24-hour high-quality service

User-friendly online submission system

Fair and swift peer-review system

Efficient typesetting and proofreading procedure

Display of the result of downloads and visits, as well as the number of cited articles

Maximum dissemination of your research work

Submit your manuscript at: http://papersubmission.scirp.org/

Or contact jbise@scirp.org 